\title{
PATH PARTITIONS AND PACKS OF ACYCLIC DIGRAPHS
}

\author{
R. Aharoni, I. Ben - Arroyo Hartman and A. J. Hoffman
}

In memory of Ernst Straus

Let $G$ be an acyclic directed graph with $|V(G)| \geq k$. We prove that there exists a colouring $\left\{C_{1}, C_{2}, \ldots, C_{m}\right\}$ such that for every collection $\left\{P_{1}, P_{2}, \ldots, P_{k}\right\}$ of $k$ vertex disjoint paths with $\left|\bigcup_{j=1}^{k} P_{j}\right|$ a maximum, each colour class $C_{i}$ meets $\min \left\{\left|C_{l}\right|, k\right\}$ of these paths. An analogous theorem, partially interchanging the roles of paths and colour classes, has been shown by Cameron [4] and Saks [17] and we indicate a third proof.

1. Introduction. Let $G=(V, E)$ be a directed graph containing no loops or multiple edges. A path $P$ in $G$ is a sequence of distinct vertices $\left(v_{1}, v_{2}, \ldots, v_{l}\right)$ such that $\left(v_{i}, v_{i+1}\right) \in E, i=1,2, \ldots, l-1$. The set of vertices $\left\{v_{1}, v_{2}, \ldots, v_{l}\right\}$ of a path $P=\left(v_{1}, v_{2}, \ldots, v_{l}\right)$ will be denoted by $V(P)$. The cardinality of $P$, denoted by $|P|$, is $|V(P)|$.

A family $\mathscr{P}$ of paths is called a path-partition of $G$ if its members are vertex disjoint and $U\{V(P): P \in \mathscr{P}\}=V$. For each nonnegative integer $k$, the $k$-norm $|\mathscr{P}|_{k}$ of a path partition $\mathscr{P}=\left\{P_{1}, \ldots, P_{m}\right\}$ is defined by

$$
|\mathscr{P}|_{k}=\sum_{i=1}^{m} \min \left\{\left|P_{i}\right|, k\right\} .
$$

A partition which minimizes $|\mathscr{P}|_{k}$ is called $k$-optimum. For example, a 1-optimum partition is a partition $P$ containing a minimum number of paths.

A partial $k$-colouring is a family $\mathscr{C}^{k}=\left\{C_{1}, C_{2}, \ldots, C_{t}\right\}$ of at most $k$ disjoint independent sets $C_{i}$ called colour classes. The cardinality of a partial $k$-colouring $\mathscr{C}^{k}=\left\{C_{1}, C_{2}, \ldots, C_{t}\right\}$ is $\mid \bigcup_{i=1}^{t} C_{i}$, and $\mathscr{C}^{k}$ is said to be optimum if $\left|\cup_{i=1}^{t} C_{i}\right|$ is as large as possible. A path partition $\mathscr{P}=$ $\left\{P_{1}, P_{2}, \ldots, P_{m}\right\}$ and a partial $k$-colouring $\mathscr{C}^{k}$ are orthogonal if every path $P_{i}$ in $\mathscr{P}$ meets $\min \left\{\left|P_{i}\right|, k\right\}$ different colour classes of $\mathscr{C}^{k}$.

Berge [2] made the following conjecture:

Conjecture 1 . Let $G$ be a directed graph and let $k$ be a positive integer. Then for every $k$-optimum path partition $\mathscr{P}$, there exists a partial $k$-colouring $\mathscr{C}^{k}$ orthogonal to $\mathscr{P}$. 
Let $\pi_{k}(G)$ be the $k$-norm of a $k$-optimum path partition in $G$, and let $\alpha_{k}(G)$ be the cardinality of an optimum partial $k$-colouring in $G$. A weaker conjecture by Linial [14] is as follows:

Conjecture 2. Let $G$ be a directed graph and let $k$ be a positive integer. Then,

$$
\alpha_{k}(G) \geq \pi_{k}(G) .
$$

If Conjecture 1 holds, then every path $P$ in a $k$-optimum path partition $\mathscr{P}$ meets at least $\min \{|P|, k\}$ vertices of some partial $k$-colouring $\mathscr{C}^{k}$. Hence, $\alpha_{k}(G) \geq \sum_{P \in \mathscr{P}} \min \{|P|, k\}=\pi_{k}(G)$, and Conjecture 2 holds.

For $k=1$, Conjecture 2 holds by the Gallai-Milgram theorem [9]. Linial [13] showed that the proof of the Gallai-Milgram theorem also yields Conjecture 1 for this case.

For transitive graphs, Conjecture 2 is given for $k=1$ by Dilworth's theorem [6], and for all $k$ by the theorem of Greene and Kleitman [10]. It is easy to deduce from it that Conjecture 1 also holds for such graphs. Linial [14] and Cameron [3] independently showed that Conjecture 2 holds for all acyclic graphs. Conjecture 1 was proved for such graphs in [1]. Cameron [4] and Saks [17] have shown that an even stronger version of Conjecture 1 holds for all acyclic graphs:

THEOREM 1. Let $G$ be a directed acyclic graph, and let $k$ be a positive integer. Then there exists a partial k-colouring $\mathscr{C}^{k}$ which is orthogonal to every k-optimum path partition $\mathscr{P}$ of $G$.

We indicate a proof of Theorem $1 \mathrm{in} \S 3$. This proof is different from the ones in [4] and [17] and was found independently.

It is possible to 'dualize' the notions of path partition and partial $k$-colouring, by interchanging the roles of 'path' and 'independent set' in the definitions and theorems above.

A colouring $\mathscr{C}$ is a partition of $V$ into disjoint independent sets. For each non-negative integer $k$, the $k$-norm $|\mathscr{C}|_{k}$ of a colouring $\mathscr{C}=$ $\left\{C_{1}, C_{2}, \ldots, C_{m}\right\}$ is defined as:

$$
|\mathscr{C}|_{k}=\sum_{i=1}^{m} \min \left\{\left|C_{i}\right|, k\right\}
$$

A colouring which minimizes $|\mathscr{C}|_{k}$ is called $k$-optimum. For example, a 1-optimum colouring is a colouring with $\chi$ colours, where $\chi$ is the chromatic number of $G$.

The analogue of a partial $k$-colouring for paths, is a path $k$-pack, defined to be a family $\mathscr{P}^{k}=\left\{P_{1}, P_{2}, \ldots, P_{t}\right\}$ of at most $k$ disjoint paths 
$P_{i}$. The cardinality of a path $k$-pack $\mathscr{P}^{k}=\left\{P_{1}, P_{2}, \ldots, P_{t}\right\}$ is $\left|\cup_{i=1}^{t} P_{t}\right|$, and $\mathscr{P}^{k}$ is optimum if $\left|\bigcup_{i=1}^{t} P_{i}\right|$ is as large as possible. A colouring $\mathscr{C}=$ $\left\{C_{1}, C_{2}, \ldots, C_{m}\right\}$ and a path $k$-pack $\mathscr{P}^{k}$ are orthogonal if every colour class $C_{i}$ in $\mathscr{C}$ meets $\min \left\{\left|C_{i}\right|, k\right\}$ different paths of $\mathscr{P}^{k}$.

As a dual analogue of Conjecture 1, we suggest the following:

Conjecture 3. Let $G$ be a directed graph and let $k$ be a positive integer. Then for every optimum path $k$-pack $\mathscr{P}^{k}$, there exists a colouring $\mathscr{C}$ orthogonal to $\mathscr{P}^{k}$.

Let $\chi_{k}(G)$ be the $k$-norm of a $k$-optimum colouring in $G$, and let $\lambda_{k}(G)$ be the cardinality of an optimum path $k$-pack in $G$. The dual of Conjecture 2 would be:

Conjecture 4. (Linial [14]). Let $G$ be a directed graph and let $k$ be a positive integer. Then,

$$
\lambda_{k}(G) \geq \chi_{k}(G)
$$

It is not difficult to see that Conjecture 3 implies Conjecture 4. For $k=1$, Conjecture 4 is given by the Gallai-Roy theorem $[7,15]$ and Conjecture 3 is also valid in this case, by the proof of the Gallai-Roy theorem.

For transitive graphs, Conjecture 4 is true by Greene's theorem [9] and Conjecture 3 can be deduced from it. Hoffman [12] and Saks [16] have independently proved Conjecture 4 for all acyclic graphs.

In this paper we prove the following stronger version of Conjecture 3 for all acyclic graphs:

THEOREM 2. Let $G$ be a directed acyclic graph and let $k$ be a positive integer. Then there exists a colouring $\mathscr{C}$ orthogonal to every optimum path k-pack $\mathscr{P}^{k}$.

2. Proof of Theorem 2. If $V$ can be covered by $k$ or fewer vertex disjoint paths, then making each vertex a colour class satisfies Theorem 2. So assume otherwise. Let $|V|=n$, and label the vertices $1,2, \ldots, n$. We shall use the linear program defined in [12]:

Let $C=\left(c_{i j}\right), i, j=0,1, \ldots, n$, be defined by

$$
\begin{aligned}
& c_{i 0}=0 \text { for all } i ; \quad c_{0 j}=1 \quad \text { for all } j>0 \\
& c_{i i}=0 \text { for all } i \\
& \text { if } i>0, j>0, \text { and } i \neq j, \text { then } c_{i j}=1 \quad \text { if }(i, j) \in E \\
& =\text { not defined if }(i, j) \notin E .
\end{aligned}
$$


Consider the transportation problem:

I.

$$
\operatorname{maximize} \sum_{\substack{i=0 \\ j=0}}^{n} c_{i j} x_{i j}
$$

where $x_{i j} \geq 0$ for all $i, j$, except that $x_{i j}$ is not defined if $i>0, j>0, i \neq j$ and $(i, j) \notin E$.

$$
\begin{gathered}
\sum_{j=0}^{n} x_{0 j}=\sum_{i=0}^{n} x_{i 0}=k \\
\sum_{j=0}^{n} x_{i j}=1 \quad \text { for } i>0 ; \quad \sum_{i=0}^{n} x_{i j}=1 \quad \text { for } j>0 .
\end{gathered}
$$

Every path $k$-pack $\mathscr{P}^{k}=\left\{P_{1}, P_{2}, \ldots, P_{t}\right\}, t \leq k$, corresponds to a feasible solution of (2.1)-(2.3), $x$, defined in the following way:

$$
x_{00}=k-t
$$

if $j>0$,

$$
\begin{aligned}
x_{0 j} & =1 & \text { if } j \text { is the start of one of } P_{1}, \ldots, P_{t} \\
& =0 & \text { otherwise. }
\end{aligned}
$$

if $i>0$,

$$
\begin{aligned}
x_{i 0} & =1 & & \text { if } i \text { is the end of one of } P_{1}, \ldots, t_{t} \\
& =0 & & \text { otherwise }
\end{aligned}
$$

if $i>0$,

$$
\begin{aligned}
x_{i i}=1 & \text { if } i \notin V\left(P_{1}\right) \cup \cdots \cup V\left(P_{t}\right) \\
=0 & \text { if } i \in V\left(P_{1}\right) \cup \cdots \cup V\left(P_{t}\right)
\end{aligned}
$$

if $i>0, j>0, i \neq j$, then

$$
\begin{array}{rlrl}
x_{i j} & =1 & \text { if }(i, j) \text { is an edge of } P_{r} \text { for some } r=1, \ldots, t \\
& =0 & & \text { otherwise. }
\end{array}
$$

It can be shown that every vertex of (2.1)-(2.3) is integral and corresponds to a path $k$-pack of $G$. Hence, an integral optimum solution of (2.1)-(2.3) corresponds to an optimum path $k$-pack, and conversely.

Consider the dual problem:

II.

$$
\min k\left(u_{0}+v_{0}\right)+\sum_{i=1}^{n} u_{i}+\sum_{j=1}^{n} v_{j}
$$


where

$$
u_{i}+v_{j} \geq c_{i j} \text { for all } i, j
$$

Complementary slackness conditions for I and II are

$$
x_{i j}>0 \Rightarrow u_{i}+v_{j}=c_{i j} \text { for all } i, j .
$$

Since the matrix of equations (2.4) is totally unimodular, the 1.p. attains its minimum at integral $u$ 's and $v$ 's. We may subtract $u_{0}$ from each $u_{i}$ and $v_{i}$, $i=0,1, \ldots, n$, to get an integral optimum solution with

$$
u_{0}=0 \text {. }
$$

We are now ready to define our colour classes. The "interesting" classes - the $S_{r}$ defined below-get their names from the values of variables. Let

$$
\begin{aligned}
& W=\left\{i>0: u_{i}+v_{\imath}=0\right\} \\
& S_{r}=\left\{i \in W: v_{\imath}=r\right\}
\end{aligned}
$$

and

$$
T_{j}=\{j\}, \quad \text { where } j \notin W .
$$

Let $s=\max \left\{v_{i} \mid i \in W\right\}$. (We shall show later that $s=v_{0}$.) We shall establish that $\mathscr{C}=\left\{S_{1}, S_{2}, \ldots, S_{s}, T_{1}, T_{2}, \ldots, T_{n}\right\}$ is a colouring of $G$ which satisfies the theorem.

To show $\mathscr{C}$ is a colouring, we need only prove that each $S_{r}$ is an independent set. Suppose not. Then there exist $i, j \in S_{r},(i, j) \in E$. But $u_{l}+v_{i}=0, u_{i}+v_{j} \geq 1$ imply $v_{j}-v_{i} \geq 1$, so $v_{i}=v_{j}=r$ is impossible.

By our stipulations at the beginning of the proof, an optimum path $k$-pack contains $k$ paths. Let $\mathscr{P}^{k}=\left\{P_{1}, P_{2}, \ldots, P_{k}\right\}$ be optimum. We must show that:

(i) each $T_{j}=\{j\}$ is on some path of $\mathscr{P}^{k}$ and

(ii) each $S_{r}$ meets all paths of $\mathscr{P}^{k}$.

To prove (i), note that $j \in T_{j}$ means $u_{j}+v_{j}>0$, implying by (2.5) that $x_{\jmath j}=0$. Since $\sum_{k} x_{\jmath k}=1$, we must have $x_{\jmath l}=1$ for some $l$, so $j$ is in some path of $\mathscr{P}^{k}$.

To prove (ii), we first observe that

$$
v_{0} \geq s \text {. }
$$

To show (2.7) we use (2.4):

$$
\begin{array}{ll}
u_{i}+v_{0} \geq c_{i 0}=0 & \forall i \in W \\
u_{i}+v_{i}=0 & \forall i \in W .
\end{array}
$$

From the last two equations we deduce that $v_{0} \geq v_{i} \forall i \in W$, and (2.7) follows. 
Next, let $P$ be a path of $\mathscr{P}^{k}$, and for ease of notation, assume the path is $(1,2, \ldots, l)$. Then

$$
x_{01}=x_{12}=\cdots=x_{l-1 l}=x_{l 0}=1 .
$$

By $(2.5), u_{0}+v_{1}=1$, so by $(2.6)$

$$
v_{1}=1 \text {. }
$$

Similarly, by (2.5), $u_{l}+v_{0}=0$, and by (2.4), $u_{l}+v_{l} \geq 0$, so

$$
v_{l} \geq v_{0} \text {. }
$$

From $u_{j}+v_{j} \geq 0$ and $u_{j}+v_{j+1}=1$ it follows that

$$
\begin{cases}\text { for } j=1,2, \ldots, l-1, \quad v_{j+1}-v_{j} \leq 1, & \text { with equality if and } \\ & \text { only if } u_{j}+v_{j}=0 .\end{cases}
$$

Together, (2.8)-(2.10) show that $S_{1}, S_{2}, \ldots, S_{v_{0}-1}$ all meet $P$. All that remains to be shown is that $S_{v_{0}}$ meets $P$.

From the proof of (2.9), we see that if $u_{l}+v_{l}=0$, then also $v_{l}=v_{0}$ and $l$ is in $S_{v_{0}}$ and in $P$. If $u_{l}+v_{l}>0$, then $v_{l}>v_{0}$. From (2.10) it follows that there is some $j<l$ with $u_{j}+v_{j}=0$ and $v_{j}=v_{l}-1 \geq v_{0}$. By (2.7), this means $v_{j}=v_{0}, j$ is in $S_{v_{0}}$ and $j$ is on $P$. This completes the proof.

Another proof of the theorem can be deduced from [5] and [11]. It is worth noting that Theorem 2 is not true for general directed graphs, as we shall show in $\S 4$.

3. An outline of a proof of Theorem 1. The proof uses ideas similar to the ones used in the proof of Theorem 2.

Let $C=\left(c_{i j}\right), i, j=0, \ldots, n$, be defined by

$$
\begin{array}{ll}
c_{i 0}=0 & \text { for all } i ; \quad c_{0 j}=k \text { for all } j, 0 \\
c_{i i}=1 & \text { for all } i>0
\end{array}
$$

if $i>0, j>0$ and $i \neq j$ then

$$
\begin{aligned}
c_{i j} & =0 \quad \text { if }(i, j) \in E \\
& =\text { not defined if }(i, j) \notin E .
\end{aligned}
$$

Consider the following linear program:

$I^{\prime}$.

$$
\operatorname{minimize} \sum_{i=0, j=0}^{n} c_{i j} x_{i j}
$$




$$
\begin{aligned}
& \left\{\begin{array}{l}
\text { where } x_{i j} \geq 0 \text { for all } i, j, \text { except that } x_{i j} \text { is not defined } \\
\text { if } i>0, j>0, i \neq j,(i, j) \notin E .
\end{array}\right. \\
& \qquad \sum_{j=0}^{n} x_{0 j}=\sum_{i=0}^{n} x_{i 0}=n \\
& \sum_{j=0}^{n} x_{i j}=1 \quad \text { for all } i>0 ; \quad \sum_{i=0}^{n} x_{i j}=1 \quad \text { for } j>0 .
\end{aligned}
$$

Let $\mathscr{P}$ be a path partition, and let $\mathscr{P}^{0}$ denote the set of all paths in $\mathscr{P}$ of cardinality at most $k$, and $\mathscr{P}^{+}$denote the set of paths in $\mathscr{P}$ of cardinality at least $k$. Paths of cardinality $k$ are assigned arbitrarily to $\mathscr{P}^{0}$ or $\mathscr{P}^{+}$. We define the following matrix

$$
\begin{aligned}
X(\mathscr{P}) & =\left(x_{\imath j}\right) \text { corresponding to } \mathscr{P}: \\
x_{00} & =n-\left|\mathscr{P}^{+}\right|
\end{aligned}
$$

if $j>0, \quad x_{0 j}=1$ if $j$ is the start of some path in $\mathscr{P}^{+}$.

$$
=0 \text { otherwise }
$$

if $i>0, \quad x_{i 0}=1 \quad$ if $i$ is the end of some path in $\mathscr{P}^{+}$.

$$
=0 \text { otherwise }
$$

if $i>0, \quad x_{i i}=1 \quad$ if $i$ belongs to some path in $\mathscr{P}^{0}$.

$=0$ otherwise

$x_{i j}=1$ if for some $P \in \mathscr{P}^{+},(i, j)$ is an edge of $P$.

$$
=0 \text { otherwise. }
$$

As in $\S 2$, it can be shown that in this correspondence, every integral optimal solution of (3.2)-(3.4) corresponds to a $k$-optimum path partition, and conversely.

Consider the dual problem.

II $^{\prime}$

$$
\begin{aligned}
& \operatorname{maximize} n\left(u_{0}+v_{0}\right)+\sum_{i=1}^{n} u_{i}+\sum_{i=1}^{n} v_{j} \\
& \text { where } u_{i}+v_{j} \leq c_{i j} \text { for all } i, j .
\end{aligned}
$$

We may assume that there exists an integral optimum solution of II' $^{\prime}$ satisfying $u_{0}=v_{0}=0, u_{\imath} \leq 0$ and $0 \leq v_{\imath} \leq k$.

We associate a partial $k$-colouring $\mathscr{C}^{k}=\left\{C_{1}, C_{2}, \ldots, C_{k}\right\}$ to such a solution in the following way. Let

$$
C_{r}=\left\{i>0: 1-u_{i}=v_{i}=r\right\} .
$$


Using the complementary slackness conditions it can be proved (as in §) that $\mathscr{C}^{k}$ is orthogonal to every $k$-optimum path partition.

4. Some counterexamples. Let $G$ be a poset, and let $\mathscr{P}$, and $\mathscr{C}^{k}$ be a path partition, and a partial $k$-colouring of $G$, respectively. Since every path $P$ in $\mathscr{P}$ meets at most $\min \{|P|, k\}$ vertices of $\mathscr{C}^{k}$, we have

$$
\left|\mathscr{C}^{k}\right| \leq \sum_{P \in \mathscr{P}} \min \{|\mathscr{P}|, k\} \text {. }
$$

If $\mathscr{P}$ and $\mathscr{C}$ are orthogonal, then equality holds and $\mathscr{P}$ is $k$-optimum and $\mathscr{C}$ is optimum. Thus, the following extension of Conjecture 1 is valid for $G$.

THEOREM 1'. For every $k$-optimum path partition $\mathscr{P}$, there exists an optimum partial $k$-colouring $\mathscr{C}^{k}$ orthogonal to $\mathscr{P}$.

However, if $G$ is not a poset, Theorem $1^{\prime}$ may not be valid, as demonstrated in the following example, for $k=1$ (see Figure 1). The set $S=\{1,3,6\}$ denotes the unique optimum independent set. $\mathscr{P}=$ $\{(1,2,3,5,6),(4)\}$ is a 1 -optimum path partition not orthogonal to $S$.

In a similar manner, the following extension of Conjecture 3 holds for all posets $G$.

THEOREM 3'. For every optimum path $k$-pack $\mathscr{P}^{k}$, there exists a $k$-optimum colouring $\mathscr{C}$ orthogonal to $\mathscr{P}^{k}$.

Theorem $3^{\prime}$ may not be valid for graphs other than posets, as shown in the following counterexample for $k=1$ (see Figure 2).

The path $P=(1,2,3,4)$ is a longest path, and $\chi(G)=3$. But any 3-colouring colours $P$ in two different colours, as shown in Figure 2.

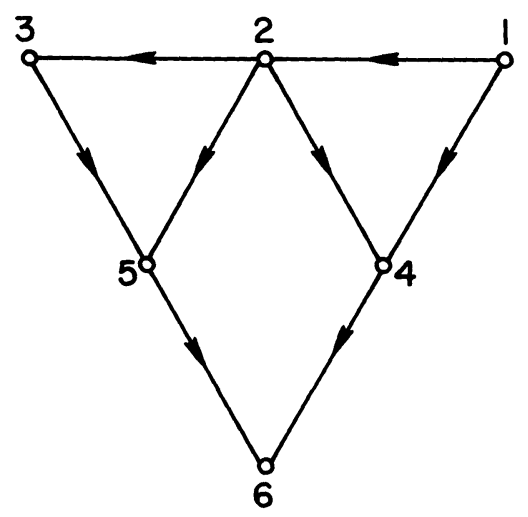

FIGURE 1 


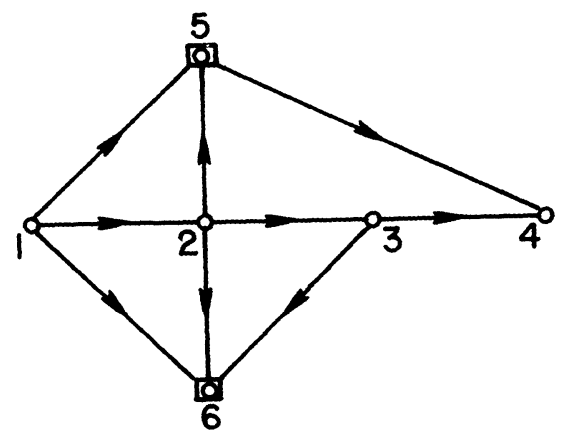

FIGURE 2

Another variant of Conjecture 1 is:

THEOREM $1^{\prime \prime}$. For every optimum partial $k$-colouring $\mathscr{C}^{k}$, there exists a path partition $\mathscr{P}$, orthogonal to $\mathscr{C}^{k}$.

It can be proved that Theorem $1^{\prime \prime}$ is valid for posets, but not in general. For $k=1$, we have the following counterexample (see Figure 3).

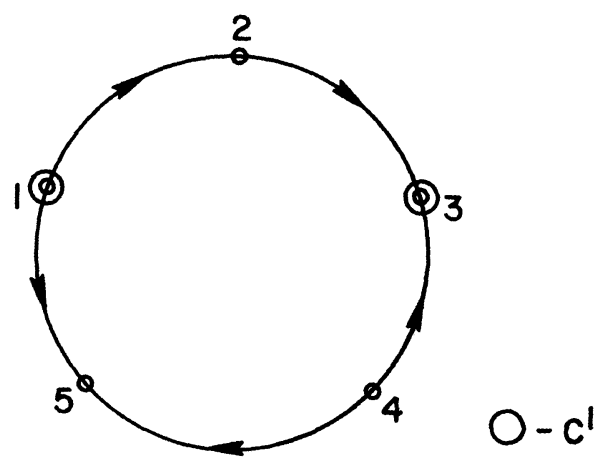

FIGURE 3

No path partition is orthogonal to $\mathscr{C}^{1}=\{(1,3)\}$ in $G$. A similar variant on Conjecture 3 is

THEOREM 3". For every k-optimum colouring $\mathscr{C}$ there exists a path k-pack orthogonal to $\mathscr{C}$.

As in Theorem 1", this theorem is valid for posets, but not for all graph, as demonstrated in Figure 4. 

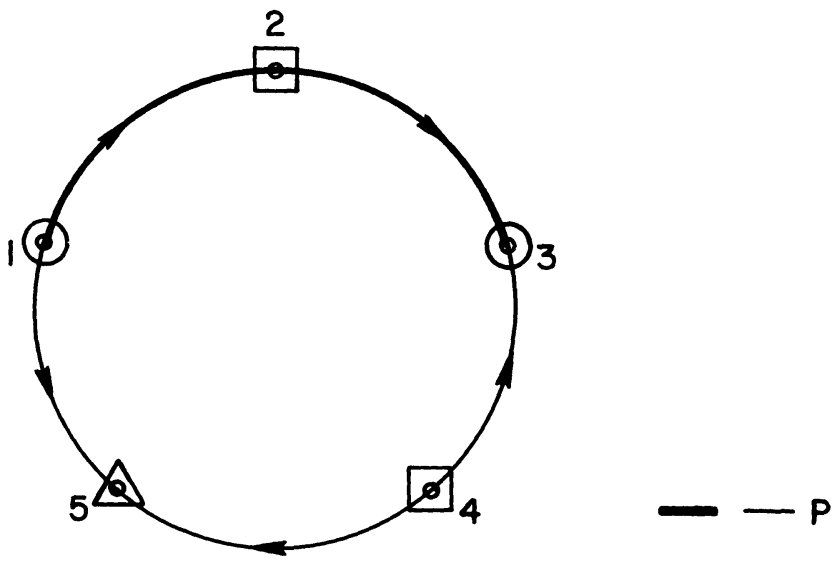

Figure 4

The path $P=(1,2,3)$ is a unique longest path but it is not orthogonal to the colouring $\mathscr{C}=\{(1,3),(2,4),(5)\}$.

Finally, we show that neither Theorem 1 nor Theorem 2 is true in general for all graphs.

Let $G=(V, E)$ be defined by (see Figure 5)

$$
V=\left\{P_{1}, P_{2}, P_{3}, P_{4}, P_{5}, Q, R\right\}
$$

and

$$
E=\left\{\left(P_{1}, P_{j}\right) \text { where } i<j\right\} \cup\left\{\left(P_{3}, Q\right),(Q, R),\left(R, P_{3}\right)\right\} \text {. }
$$

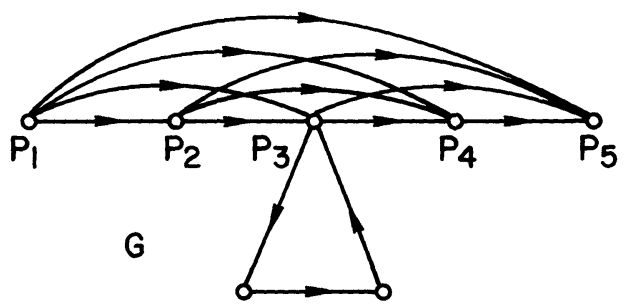

FIGURE 5

It can be verified that for any maximum independent set $S$ in $G$, there exists a path partition which is not orthogonal to $S$. Also, there is no way of colouring $G$ so that all longest paths (there are three of them) meet all colours. Hence $G$ serves as a counterexample for $k=1$ for Theorem 1 as well as for Theorem 2, when considered for general graphs. 


\section{REFERENCES}

[1] R. Aharoni and I. Ben-Arroyo Hartman, On k-optimal path partitions of acyclic digraphs, Technion preprint series NO MT 624, November 1983.

[2] C. Berge, $k$-optimal partitions of a directed graph, Europ. J. Combinatorics, 3 (1982), 97-101.

[3] K. B. Cameron, Polyhedral and algorithmic ramifications of antichains, $\mathrm{Ph} . \mathrm{D}$. thesis, University of Waterloo, 1982.

[4] On k-optimum dipath partitions and partial k-colourings of acyclic digraphs, preprint.

[5] G. B. Dantzig and A. Hoffman, On a theorem of Dilworth, "Contributions to linear inequalities and related topics", Ann. Math. Studies, 38.

[6] R. P. Dilworth, A decomposition theorem for partially ordered sets, Ann. of Math., $\mathbf{5 1}$ (1950), (6).

[7] T. Gallai, On Directed Paths and Circuits, in: Theory of Graphs, (eds. P. Erdos and G. Katona), Academic Press, New York, 1968, pp. 115-118.

[8] T. Gallai and A. N. Milgram, Verallgemeinerung eines Graphentheorischen Satzes von Redei, Acta Sci. Math., 21 (1960), 181-186.

[9] C. Greene, Some partitions associated with a partially ordered set, J. Combinatorial Theory, Ser. A, 20 (1976), 69-79.

[10] C. Greene and D. J. Kleitman, The structure of Sperner k-families, J. Combinatorial Theory, Ser. A, 20 (1976), 41-68.

[11] Groflin and A. J. Hoffman, Lattice polyhedra II: Generalization, construction and examples, Ann. Discrete Math., 15 (1982), 189-203.

[12] A. J. Hoffman, Extending Greene's theorem to directed graphs, J. Combinatorial Theory, Ser. A, 34 (1983), 102-107.

[13] N. Linial, Covering digraphs by paths, Discrete Math., 23 (1978), 257-272.

[14] Extending the Greene-Kleitman theorem to directed graphs, J. Combinatorial Theory, Ser. A, 30 (1981), 331-334.

[15] B. Roy, Nombre chromatique et plus longs chemins, Rev. Fr. Automat. Informat., 1 (1967), 127-132.

[16] M. Saks, A short proof of the k-saturated partitions, Adv. in Math., 33 (1979), 207-211.

[17] _ Some integer sequences associated with combinatorial structures, in preparation.

Received June 6, 1984. This forms part of the second author's research towards a Ph.D., supervised by J. A. Bondy. This work was done while the third author was visiting the IBM-Scientific Center, Haifa, Israel.

IBM-Thomas J. Watson Research Center

YORKTOWN HeIGHTS, NY 10598 
